\title{
Signature Sequence Selection for CDMA Systems with Multiple Receiver Antennas
}

\author{
Semih Serbetli and Aylin Yener \\ Electrical Engineering Department \\ The Pennsylvania State University \\ University Park, PA 16802 \\ serbetli@psu.edu_yener@ee.psu.edu
}

\begin{abstract}
We consider the problem of signature sequence selection for the uplink of CDMA systems with multiple receiver antennas. We consider the sum capacity, the systemwide MSE, and the effective total squared correlation as performance metrics and formulate iterative algorithms that improve these metrics. The convergence analysis of the algorithms is given and the numerical evidence that supports the analysis is presented along with our observations related to the resulting optimum signature sets.
\end{abstract}

\section{INTRODUCTION}

The rapid growth of wireless services demands that next generation systems support higher data rates reliably. CDMA is a promising candidate to meet this challenge. Interference suppression techniques to improve the performance of single antenna CDMA systems have been studied extensively [1]. Recent studies indicate that using multiple antennas at the receiver can further improve the performance by providing interference suppression not only by exploiting the temporal structure of the received signal, but also the spatial signal space dimensions [2]. The potential of using multiple receiver antennas and the design of combined spatial-temporal receivers that realizes this potential are well understood [3].

Performance of a CDMA system is highly influenced by the signature sequences of the users. Transmitter based interference mitigation, termed interference avoidance, has been investigated recently [4]-[7]. For single antenna systems, signature sets that maximize the information theoretic sum capacity of synchronous CDMA systems are identified in [4], [5]. A number of iterative algorithms that converge to the global optimum signature sequence sets have been constructed considering the systemwide MSE, the sum capacity, and the total squared cross correlation as performance metrics [6]-[9].

Transmitter precoding (shaping) has been studied for various multiuser systems such as multipath CDMA channels [10] and space division multiple access (SDMA) systems [11] for scenarios where one symbol per user is transmitted. Iterative algorithms that find sum capacity maximizing transmitter covariance matrices, when multiple symbols of each user are multiplexed, are studied for multiuser MIMO systems [12]. Reference [13] studies the transmitter precoding problem for multiuser MIMO systems with system-wide MSE as the performance metric.
In this paper, we investigate the problem of selecting the best possible signature sequence set for a CDMA system when the (base station) receiver employs multiple antennas. The presence of multiple receive antennas provides additional signal space dimensions that potentially improves the performance of the multiple access channel with an intelligent choice of transmitters. We note that, the transmitter optimization problem in this case, is far from a straight forward extension of the single antenna case [4], [5], or the case of multiple antennas with independent fading [14]. Specifically, we consider the case of given channel realizations. In this case, we have fixed spatial signatures for users, which we show to amount to constraining the temporal-spatial signal space from which the transmitters ought to be selected.

We consider first, the sum capacity and the system-wide MSE as our performance criteria. We next formulate the signature selection problem as the choice of the set of signatures that would closely approximate the optimum signature sets with no channel constraints. We show that such a least squares fitting approach reduces to the minimization of the effective total squared correlation. We observe that, in contrast with the single receive antenna case, the optimization of each of these objectives, with the constraints introduced by the channel realizations, may result in different optimum signature sequence sets. For each of these performance criteria, we construct iterative algorithms that improve the performance at each step and provide evidence that the corresponding optimum case is achieved in each case. We also include our observations regarding the resulting optimum signature sets. It must be noted that for the CDMA signature sequence selection problem with multiple receive antennas, three regions of interest for system load arise. Not surprisingly, when the number of users is less than the processing gain, orthogonal signature sequence sets are optimum; and when the number of users is larger than the product of the processing gain and the number of antennas, the optimum sequences try to approach WBE sets. For the intermediate region where the number of users is in between the two numbers, a region unique to the multiple receive antenna problem, we observe that signature sequence space is partitioned between spatially highly correlated users, that is a known suboptimum solution for the single antenna case [6], may turn out to be optimum. We provide analytical 
reasoning behind this result by an example of a small system. We also provide extensive numerical results for convergence and the performance of the iterative algorithms optimizing the several criteria considered.

\section{SySTEM MODEL}

We consider the uplink of a synchronous $K$-user DS-CDMA system with processing gain $N$. We assume $L$ observations of the transmitted signal are received resulting from antenna arrays at the base station, or cooperation of different base stations, or both. Similar to the notation in [3], the signal received at the $i$ th antenna is

$$
r_{i}(t)=\sum_{j=1}^{K} \sqrt{P_{j}} b_{j} s_{j}(t) h_{i j}+n_{i}(t)
$$

where $P_{j}, b_{j}$ and $s_{j}(t)$ are the transmit power, bit and the signature of user $j$, respectively, and $h_{i j}$ represents the uplink channel gain of user $j$ to the $i$ th receive antenna. The white Gaussian noise at the $i$ th receive antenna is denoted as $n_{i}(t)$. We assume that the signal of each user is received with unit total power, i.e., $\sum_{i=1}^{L} P_{j}\left\|h_{i j}\right\|^{2}=1$. For clarity of notation, we denote the joint effect of the transmit power and channel gain of user $j$ to $i$ th receive antenna as $a_{i j}=\sqrt{P_{j}} h_{i j}$ which forms a unit norm spatial signature $\mathbf{a}_{j}=\left[a_{1 j}, a_{2 j}, \cdots, a_{L j}\right]^{T}$. Chip matched filtering the received signal and sampling at the chip rate, we have the discrete representation of the signal at the output of receive antenna $i$ as

$$
\mathbf{r}_{i}=\sum_{j=1}^{K} b_{j} \mathbf{s}_{j} a_{i j}+\mathbf{n}_{i}=\mathbf{S} \mathbf{A}_{i} \mathbf{b}+\mathbf{n}_{i}
$$

where $\mathbf{S}=\left[\mathbf{s}_{1} \mathbf{s}_{2} \cdots \mathbf{s}_{K}\right]$ is the signature sequence matrix, $\mathbf{A}_{i}=\operatorname{diag}\left\{a_{i 1}, a_{i 2}, \cdots a_{i K}\right\}$ is the diagonal gain matrix for the $i$ th receive antenna, $\mathbf{b}=\left[b_{1}, b_{2}, \cdots, b_{K}\right]^{T}$ is the bit vector of all users, and $\mathbf{n}_{i}$ is the white Gaussian noise with variance $\sigma^{2}$ at the $i$ th receive antenna. Stacking all the signal vectors of each antenna, the received signal can be represented in a vector form:

$$
\mathbf{r}=\mathbf{Q b}+\mathbf{n}
$$

with $\mathbf{Q}=\left[\begin{array}{llll}\mathbf{A}_{1}^{T} \mathbf{S}^{T} & \mathbf{A}_{2}^{T} \mathbf{S}^{T} & \cdots & \mathbf{A}_{L}^{T} \mathbf{S}^{T}\end{array}\right]^{T}$. We define the effective signature of user $i$ as the $i$ th column of $\mathbf{Q}$, i.e.,

$$
\mathbf{q}_{i}=\left[a_{1 i} \mathbf{s}_{i}^{T} a_{2 i} \mathbf{s}_{i}^{T} \cdots a_{L i} \mathbf{s}_{i}^{T}\right]^{T}
$$

\section{Performance Criteria and Update Mechanisms}

Our aim in this section is to formulate the three system performance criteria to be optimized by selecting the right signature sequences and develop iterative algorithms that arrive at the solution of each of the optimization problems.

\section{A. System Performance Criteria}

Previous work showed that the information theoretic sum capacity of a system with a given signature matrix $\mathbf{Q}$ is [4]

$$
C_{\text {sum }}=\frac{1}{2} \log \left[\operatorname{det}\left(\mathbf{I}_{N L}+\sigma^{-2} \mathbf{Q} \mathbf{Q}^{\dagger}\right)\right]=\prod_{i=1}^{N L}\left(\frac{\lambda_{i}}{\sigma^{2}}+1\right)
$$

where $\left\{\lambda_{i}\right\}$ 's are the eigenvectors of $\mathbf{Q} \mathbf{Q}^{\dagger}$. It is important to note that this formulation assumes known spatial signatures, that is a given realization of the vector channel state.

The systemwide MSE, similarly, can be represented in terms of the eigenvalues of $\mathbf{Q Q}^{\dagger}$ as [13]

$$
\begin{aligned}
\mathrm{MSE} & =K-N L+\sigma^{2} \operatorname{tr}\left\{\left(\mathbf{Q} \mathbf{Q}^{\dagger}+\sigma^{2} \mathbf{I}\right)^{-1}\right\} \\
& =K-N L+\sigma^{2} \sum_{i=1}^{N L} \frac{1}{\lambda_{i}+\sigma^{2}}
\end{aligned}
$$

The use of multiple receiver antennas avails additional signal space dimensions subject to the spatial signatures of the users. The best case scenario is when the channel realizations of the users do not prevent the joint spatial-temporal signatures to achieve the unconstrained optimum, i.e., orthogonal signature sequences when the number of users, $K$, is less than or equal to $N L$, and WBE sequences otherwise. It is conceivable then to try to find the signature sets that are as close as possible to the unconstrained optimum. In an effort to do this, we use the least squares fitting approach. The cost function to be minimized for $K \leq N L$ is

$f(\mathbf{S})=\left\|\mathbf{Q}^{\dagger} \mathbf{Q}-\mathbf{I}\right\|^{2}=\operatorname{tr}\left\{\left(\mathbf{Q} \mathbf{Q}^{\dagger}\right)^{2}\right\}-K=\sum_{i=1}^{N L} \lambda_{i}^{2}-K$

and, the squared Frobenius norm of the difference between $\mathbf{Q Q}^{\dagger}$ and $\mathbf{Q}_{W B E} \mathbf{Q}_{W B E}^{\dagger}=\frac{K}{N L} \mathbf{I}$ for $K>N L$ :

$$
\begin{aligned}
f(\mathbf{S}) & =\left\|\mathbf{Q} \mathbf{Q}^{\dagger}-\frac{K}{N L} \mathbf{I}\right\|^{2}=\operatorname{tr}\left\{\left(\mathbf{Q} \mathbf{Q}^{\dagger}\right)^{2}\right\}-\frac{K^{2}}{N L} \\
& =\sum_{i=1}^{N L} \lambda_{i}^{2}-\frac{K^{2}}{N L}
\end{aligned}
$$

In (8) and (9), $\operatorname{tr}\left\{\left(\mathbf{Q} \mathbf{Q}^{\dagger}\right)^{2}\right\}$ represents the total squared correlation of the joint spatial-temporal signature sequences that will be denoted as ETSC for the sequel. Thus, the least squares fitting approach reduces to minimization of the ETSC.

The MSE and ETSC are Schur-convex functions of the eigenvalues of $\mathbf{Q Q}^{\dagger}$, and the sum capacity is a Schur-concave function of the same [15]. Each objective can be improved if $\mathbf{Q Q}^{\dagger}$ is updated such that the eigenvalues of the new $\mathrm{QQ}^{\dagger}$ is majorized by the old [15]. For single antenna CDMA systems, this results in WBE sequences for all three criteria [8]. On the other hand, in the case of multiple receive antennas, with the resulting effective signatures in (4), the optimization of the objectives (5), (7) and (8)/(9) has to be done in the signal space constrained by the particular channel realization. Consequently, they are no longer guaranteed to be optimized by the same set of signatures.

\section{B. Iterative Update Mechanisms}

The sum rate in (5) can be expressed as

$$
C_{\text {sum }}=\frac{1}{2} \log \left[\operatorname{det}\left(\mathbf{I}_{N L}+\sigma^{-2} \sum_{i=1}^{K} \mathbf{q}_{i} \mathbf{q}_{i}^{\dagger}\right)\right]
$$




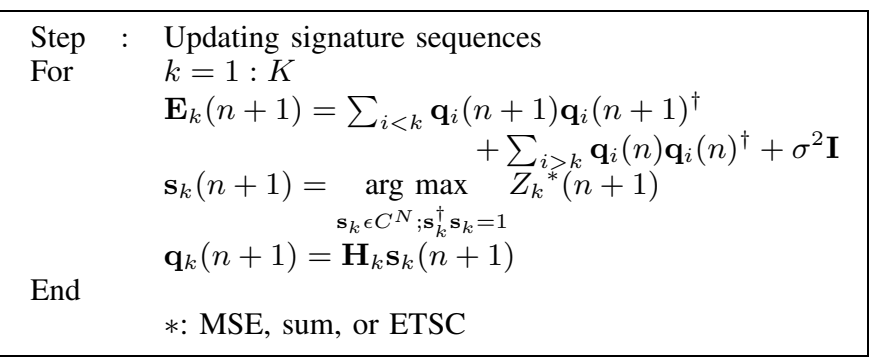

TABLE I

Iterative Signature SEQUence Update Algorithm

where the effective spatial-temporal signature of user $i, \mathbf{q}_{i}$, is given in (4). Substituting $\mathbf{q}_{i}=\mathbf{H}_{i} \mathbf{s}_{i}$ with

$$
\mathbf{H}_{i}=\left[\begin{array}{llll}
a_{i 1} \mathbf{I} & a_{i 2} \mathbf{I} & \cdots & a_{i L} \mathbf{I}
\end{array}\right]^{T}
$$

in (11), we have

$$
C_{\text {sum }}=\frac{1}{2} \log \left[\operatorname{det}\left(\mathbf{I}_{N L}+\sigma^{-2} \sum_{i=1}^{K} \mathbf{H}_{i} \mathbf{s}_{i} \mathbf{s}_{i}^{\dagger} \mathbf{H}_{i}^{\dagger}\right)\right]
$$

From user $k$ 's perspective sum capacity is expressed in as:

$$
\begin{aligned}
C_{\text {sum }} & =\pi_{k}+\frac{1}{2} \log \left(1+\mathbf{s}_{k}^{\dagger} \mathbf{H}_{k}^{\dagger} \mathbf{E}_{k}^{-1} \mathbf{H}_{k} \mathbf{s}_{k}\right) \\
& =\pi_{k}+\frac{1}{2} \log \left(1+Z_{k}^{\text {sum }}\right)
\end{aligned}
$$

where $\mathbf{E}_{k}=\sum_{i \neq k}^{K} \mathbf{H}_{i} \mathbf{s}_{i} \mathbf{s}_{i}^{\dagger} \mathbf{H}_{i}^{\dagger}+\sigma^{2} \mathbf{I}_{N L}$ is the interference covariance matrix of user $k, \pi_{k}$ represents the terms independent of user $k$, and $Z_{k}{ }^{\text {sum }}$ is the term denoting the effect of user $k$ on the sum capacity.

Similarly, the systemwide MSE can be expressed in terms of user $k$ 's parameters as follows:

$$
\begin{aligned}
\mathrm{MSE} & =\gamma_{k}-\sigma^{2}\left(\frac{\mathbf{s}_{k}^{\dagger} \mathbf{H}_{k}^{\dagger} \mathbf{E}_{k}^{-2} \mathbf{H}_{k} \mathbf{s}_{k}}{1+\mathbf{s}_{k}^{\dagger} \mathbf{H}_{k}^{\dagger} \mathbf{E}_{k}{ }^{-1} \mathbf{H}_{k} \mathbf{s}_{k}}\right) \\
& =\gamma_{k}-\sigma^{2} Z_{k}^{\mathrm{MSE}}
\end{aligned}
$$

with $\mathbf{E}_{k}$ defined as before, $\gamma_{k}$ represents the terms independent of user $k$, and $Z_{k}{ }^{\mathrm{MSE}}$ is the term denoting the effect of user $k$ on the MSE.

ETSC can be expressed in terms of user $k$ 's parameters as

$$
\begin{aligned}
\mathrm{ETSC} & =\sum_{i=1}^{K} \sum_{j=1}^{K}\left\|\mathbf{s}_{i}^{\dagger} \mathbf{H}_{i}^{\dagger} \mathbf{H}_{j} \mathbf{s}_{j}\right\|^{2} \\
& =\zeta_{k}+2 \mathbf{s}_{k}^{\dagger} \mathbf{H}_{k}^{\dagger} \mathbf{E}_{k} \mathbf{H}_{k} \mathbf{s}_{k}=\zeta_{k}-Z_{k}^{\mathrm{ETSC}}
\end{aligned}
$$

with $\mathbf{E}_{k}$ defined as before, and $\zeta_{k}$ represents the terms independent of user $k$, and $Z_{k}{ }^{\text {ETSC }}$ is the term denoting the effect of user $k$ on the ETSC.

Iterative algorithms that improve the sum capacity, the MSE, and the ETSC at each step can be devised by optimizing the signature of one user, say user $k, \mathbf{s}_{k}$, at each step. From the perspective of user $k$, the performance metric selected can be improved by choosing $\mathbf{s}_{k}$ to maximize $Z_{k}{ }^{\text {sum }}, Z_{k}{ }^{\mathrm{MSE}}$ or $Z_{k}{ }^{\mathrm{ETSC}}$. This is accomplished by choosing $\mathbf{s}_{k}$ to be the maximum eigenvalued eigenvector of $\mathbf{H}_{k}^{\dagger} \mathbf{E}_{k}^{-1} \mathbf{H}_{k}$ for maximizing the sum capacity, the maximum generalized eigenvalued eigenvector of $\mathbf{H}_{k}^{\dagger} \mathbf{E}_{k}{ }^{-2} \mathbf{H}_{k}$ and $\mathbf{I}+\mathbf{H}_{k}^{\dagger} \mathbf{E}_{k}{ }^{-1} \mathbf{H}_{k}$ for minimizing the systemwide MSE, and the minimum eigenvalued eigenvector of $\mathbf{H}_{k}^{\dagger} \mathbf{E}_{k} \mathbf{H}_{k}$ for minimizing the ETSC at each step.

In the case of a single receive antenna, the channel matrices are identity, and the updates described above for each criterion are identical. However, this is no longer the case for CDMA systems with multiple receive antennas. The updates for each criterion in general result in different sequences at each step, and there is no longer a guarantee that they will converge to the same set. In addition, the objective functions given by (5), (7), and (18) may now have local minima due to the fact that the joint spatial-temporal signature sequences are not the eigenvectors of the received signal covariance matrix, and the signature space of users is constrained by the spatial structure. Nevertheless, to improve the system performance of the system, we can devise iterative algorithms that monotonically improve each of the system performance criterion by using (15), (17) and (19). In each case we would maximize the corresponding term, $Z_{k}{ }^{\text {sum }}, Z_{k}^{\mathrm{MSE}}$, or $Z_{k}{ }^{\mathrm{ETSC}}$ for each user $k$ and iterate over users. The algorithms iterate over the users improving the system performance at each step. The outline of the algorithm is presented in Table I where $Z_{k}{ }^{*}$ should be replaced by one of the $Z_{k}^{\text {sum }}, Z_{k}^{\mathrm{MSE}}$, and $Z_{k}^{\text {ETSC }}$ according to the performance criterion chosen. Each algorithm produces monotonic sequences. In addition, the sum capacity is upper bounded by the sum rate that would be achievable if the effective signatures were unconstrained, the MSE is lower bounded by 0 , and ETSC is lower bounded by K. Therefore each algorithm is convergent.

\section{OBSERVATIONS}

In this section, we present our observations related to the proposed signature sequence updates. The use of multiple receivers increases the dimension of the signal space, and introduces a new region of number of users that we need to examine carefully. When the number of users is larger than the processing gain $N$, but less than the product of the processing gain and the number of antennas $N L$, the system is overloaded for the temporal dimensions, but can be underloaded for the joint spatial-temporal signal space. It has been observed that both the greedy signature sequence update mechanism, and the MMSE signature sequence update mechanism proposed in [7] have suboptimum fixed points. These suboptimum points are identified as the signature sequences that partition the signal space into orthogonal subsets. Reference [9] proved that these suboptima are unstable. While one may be tempted to believe the same will hold for the multiple antenna case, we observed that the iterative updates of the proposed algorithms may result in stable fixed points that are temporally partitioned signature sequences when the number of users is less than $N L$ and larger than $N$. The iterative updates converge to signature sequences that orthogonally partition the signal space in such a way that the spatially highly correlated users use orthogonal signatures. These are the cases when the system is underloaded for the joint spatial-temporal space and the algorithms in this case try 


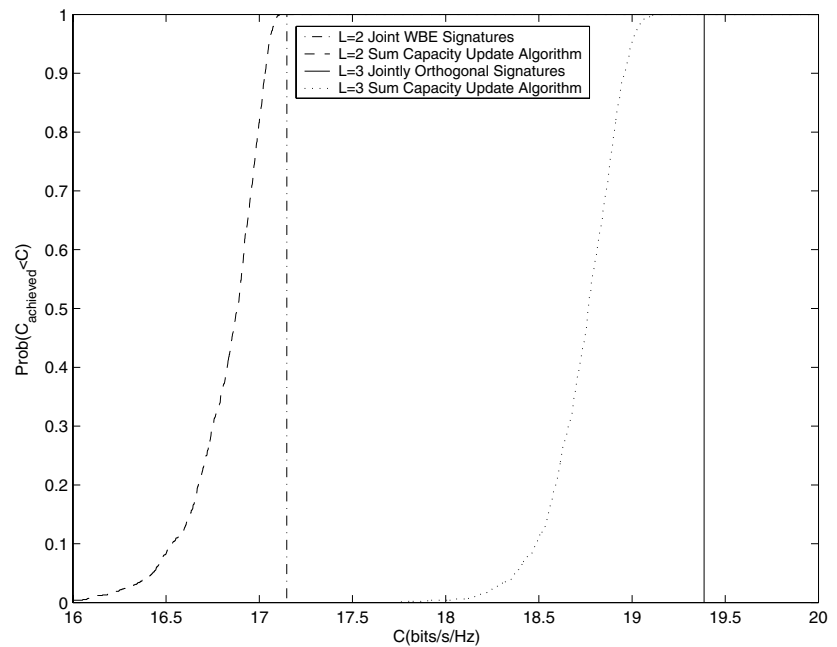

Fig. 1. $K=15$ user multiple receiver CDMA system with $N=6$ and $L=2,3$. Sum capacity CDF analysis

to orthogonalize the joint spatial-temporal signature sequences of all users. When the number of users is larger than the dimensions of the available spatial-temporal signature space then the system is strictly overloaded, and we observe that the algorithms try to "approach" the joint spatial-temporal WBE sequences optimizing the associated cost functions.

To validate our observations, let us consider a 3 user CDMA system with 2 multiple receivers and a processing gain 2 with ETSC as the performance metric. ETSC of such a system is

$$
\begin{aligned}
\mathrm{ETSC} & =\sum_{i=1}^{K} \sum_{j=1}^{K}\left\|\mathbf{s}_{i}^{\dagger} \mathbf{H}_{i}^{\dagger} \mathbf{H}_{j} \mathbf{s}_{j}\right\|^{2}=\sum_{i=1}^{K} \sum_{j=1}^{K} \delta_{i j}^{2}\left\|\mathbf{s}_{i}^{\dagger} \mathbf{s}_{j}\right\|^{2} \\
& =\sum_{i=1}^{K} \sum_{j=1}^{K} P_{e i} P_{e j}\left\|\mathbf{s}_{i}^{\dagger} \mathbf{s}_{j}\right\|^{2}-\sum_{j=1}^{K}\left(1-P_{e j}^{2}\right)
\end{aligned}
$$

where $\delta_{i j}=\left\|\mathbf{a}_{i}^{\dagger} \mathbf{a}_{j}\right\|$ and $P_{e j}=\frac{\delta_{i j} \delta_{j k}}{\delta_{i k}}$. Observe that the second term in (21) does not affect the minimization of ETSC and the first term in (21) is in the form of ETSC of a 3 user CDMA system with received powers, $\left\{P_{e j}\right\}$. Thus, depending on the effective power values $\left\{P_{e j}\right\}$, some users may be oversized, and orthogonal partitioning of the signal space can be optimum [5]. Thus, even if the actual received powers of the users are equal, $\left\{P_{e j}\right\}$ s may result in oversized users due to the cross correlation of spatial signatures, and orthogonal partitioning of the signature space may be optimum.

We note that as the number of receivers increases, the probability of a user finding another user with an arbitrarily low spatial cross correlation is increased. However, for the orthogonal partitioning of the signature space to be optimum, the users should share the signatures with a larger group of users up to $L$ users. Thus, for a CDMA system with fixed $N L$, even if the dimensions of joint spatial-temporal signal space are the same, it is more likely that the system with larger $N$ has optimum signatures that are orthogonally partitioned. This is due to the fact that decreasing the number of receivers increases the probability of a user to find a smaller

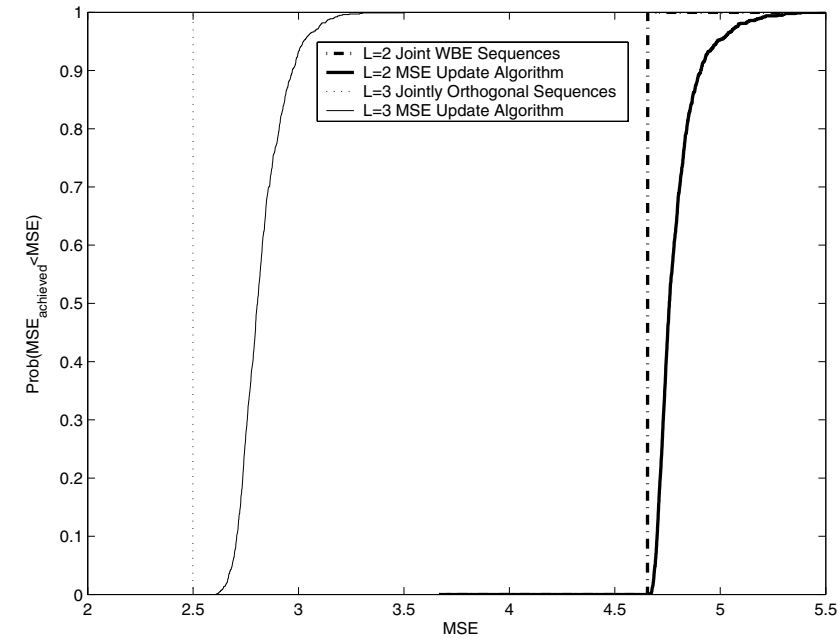

Fig. 2. $K=15$ user multiple receiver CDMA system with $N=6$ and $L=2,3$. MSE CDF analysis

group of users with an arbitrarily low spatial cross correlation and increases the portion of the joint signal space that is controllable.

\section{Numerical Results}

In this section, we present numerical results related to the performance of the proposed algorithms and the observations we outlined in the previous section. We also compare the performance of the resulting transmission schemes with, randomly generated WBE signature sequences to investigate the benefit gained by exploiting the spatial structure of the system, and the optimum (unconstrained) signature sequences for a processing gain of $N L$ that forms an upper bound for the performance.

The simulations are performed for a multiple receiver CDMA system with a processing gain $N=6$. Each user is equipped with single antenna whereas the common receiver is equipped with multiple antennas as stated for different cases. The channels are realizations of a flat fading channel where all links are independent and identically distributed complex Gaussian. Perfect power control is assumed. The received SNR of each user is $7 \mathrm{~dB}$. CDF curves for the sum capacity, systemwide MSE and ETSC of the resulting signature selection schemes obtained by simulating 10000 channel realizations are presented. We consider a multiple antenna CDMA system with $K=15$ for number of receive antennas, 2 and 3. It is observed that each system performance criterion is improved at each iteration, and converges to a fixed point for each case. Figures 1, 2 and, 3 show the $\mathrm{CDF}$ of the associated performance criterion, and the optimum performance of unconstrained signatures. As number of receive antennas is increased, better system performance can be achieved as expected, since each added receive antenna increases the spatial diversity of the users.

For comparison, a multiple antenna CDMA system using a randomly generated WBE signature set is evaluated in terms of sum capacity, MSE and ETSC for a given set of 


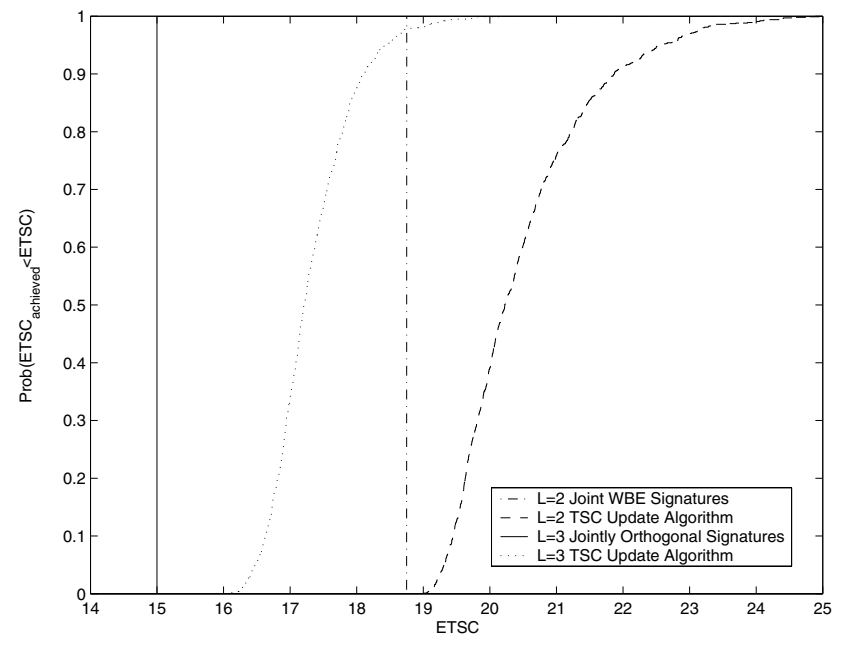

Fig. 3. $K=15$ user multiple receiver CDMA system with $N=6$ and $L=2,3$. ETSC CDF analysis

\begin{tabular}{|l|c|c|c|}
\hline Scheme & Sum Capacity & MSE & ETSC \\
\hline \hline Temporal WBE & 15.2738 & 5.7265 & 26.4114 \\
\hline Sum Capacity Update & $\mathbf{1 6 . 8 0 5 9}$ & 4.7931 & 20.6505 \\
\hline MSE Update & 16.8036 & $\mathbf{4 . 7 9 2 2}$ & 20.7146 \\
\hline ETSC Update & 16.7989 & 4.7991 & $\mathbf{2 0 . 6 1 0 2}$ \\
\hline Joint WBE Sequences & 17.1479 & 4.6552 & 18.7500 \\
\hline
\end{tabular}

TABLE II

COMPARISON OF ALGORITHMS FOR L $=2$

realization of channels for both $L=2$ and $L=3$. We also present the evolution of the ETSC in Figure 4. The proposed algorithms are observed to result in better signature sets than the randomly generated WBE signature sets. The performance of the optimum signature sequences with processing gain $N L$ which is an upper bound for all the three performance criteria is also evaluated. The comparison of the resulting signature sets are given in Table II and III.

\section{CONCLUSION}

In this paper, we investigated the problem of signature sequence selection for a multiple receiver CDMA system. We considered several performance criteria, namely, the sum capacity, total MSE, and a least squares fitting approach that reduces to effective TSC minimization. We investigated the relationship between the number of receive antennas and the performance of the system. We observed that the performance of the system can be enhanced by exploiting the spatial structure of the system, the resulting structures are not trivial extensions of the single antenna case, and deserve our continuing attention.

\section{REFERENCES}

[1] S. Verdú. Multiuser Detection. Cambridge University Press, 1998.

[2] A. F. Naguib, A. J. Paulraj, and T. Kailath. Capacity improvement with base-station antenna arrays in cellular CDMA. IEEE Transactions on Vehicular Technology, 43(3):691-698, August 1994.

[3] A. Yener, R. D. Yates, and S. Ulukus. Combined multiuser detection and beamforming for CDMA systems: Filter structures. IEEE Transactions on Vehicular Technology, 51(5):1087 - 1095, Sept. 2002.

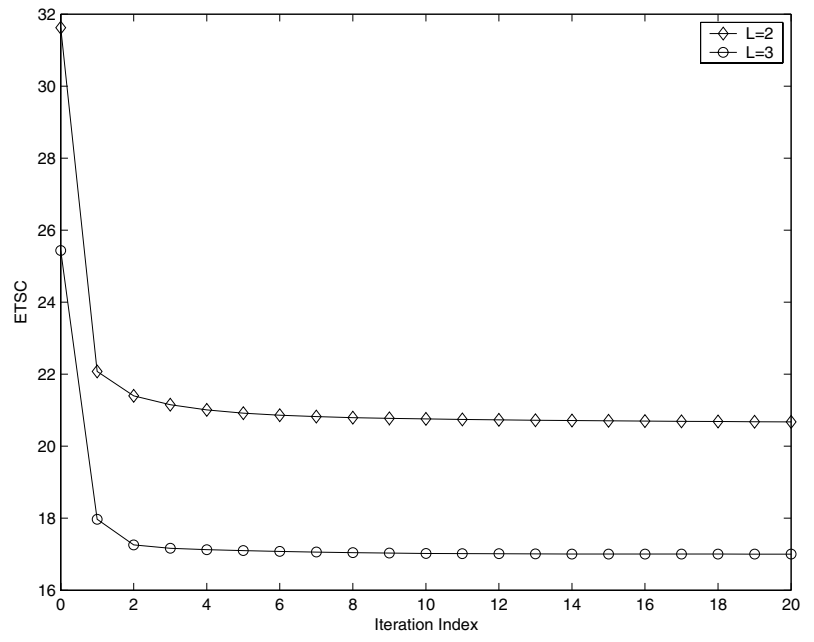

Fig. 4. $K=15$ user multiple receiver CDMA system with $N=6$ and $L=2,3$. ETSC analysis of the ETSC update at each iteration

\begin{tabular}{|l|c|c|c|}
\hline Scheme & Sum Capacity & MSE & ETSC \\
\hline \hline Temporal WBE & 17.2646 & 3.8428 & 21.9786 \\
\hline Sum Capacity Update & $\mathbf{1 8 . 8 7 2 5}$ & 2.75 & 17.0054 \\
\hline MSE Update & 18.8689 & $\mathbf{2 . 7 4 7 8}$ & 17.0909 \\
\hline ETSC Update & 18.8455 & 2.7736 & $\mathbf{1 6 . 9 9 8 3}$ \\
\hline Jointly Orth. Sequences & 19.3872 & 2.5000 & 15 \\
\hline \multicolumn{4}{|c}{ TABLE III }
\end{tabular}

COMPARISON OF ALGORITHMS FOR L $=3$

[4] M. Rupf and J. L. Massey. Optimum sequence multisets for synchronous code-division multiple-access channels. IEEE Transactions on Information Theory, 40(4):1261-1266, July 1994.

[5] P. Viswanath and V. Anantharam. Optimal sequences and sum capacity of synchronous CDMA systems. IEEE Transactions on Information Theory, 45(6):1984-1991, September 1999.

[6] S. Ulukus and R. D. Yates. Iterative construction of optimum signature sequence sets in synchronous CDMA systems. IEEE Transactions on Information Theory, 47(5):1989-1998, July 2001.

[7] C. Rose, S. Ulukus, and R.D. Yates. Wireless systems and interference avoidance. IEEE Transactions on Wireless Communications, 1(3):415428, July 2002.

[8] S. Ulukus and A. Yener. Iterative transmitter and receiver optimization for CDMA networks. IEEE Transactions on Wireless Communications, to appear. Available at http://labs.ee.psu.edu/faculty/yener/publications3.html/.

[9] P. Anigstein and V. Anantharam. Ensuring convergence of the MMSE iteration for interference avoidance to the global optimum. IEEE Transactions on Information Theory, 49(4):873 - 885, April 2003.

[10] G.S. Rajappan and M.L. Honig. Signature sequence adaptation for DSCDMA with multipath. IEEE Journal on Selected Areas in Communications, 20(2):384 - 395, February 2002.

[11] J.-H. Chang, F. Rashid-Farrokhi, and L. Tassiulas. Joint transmitter receiver diversity for efficient space division multiple access. IEEE Transactions on Wireless Communications, 1(1):16-26, Jan. 2002.

[12] W. Yu, W. Rhee, S. Boyd, and J.M. Cioffi. Iterative water-filling for Gaussian vector multiple access channels. IEEE Transactions on Information Theory, 50(1):145-152, Jan. 2004.

[13] S. Serbetli and A. Yener. Transceiver optimization for multiuser MIMO systems. IEEE Transactions on Signal Processing, 52(1):214 - 226, Jan. 2004.

[14] S. V. Hanly and D. N. C. Tse. Resource pooling and effective bandwidths in CDMA systems with multiuser receivers and spatial diversity. IEEE Transactions on Information Theory, 47(4):1328-1351, May 2001.

[15] A. Marshall and I. Olkin. Inequalities: Theory of Majorization and Its Applications. New York: Academic, 1979. 